\title{
Frequency of Cocaine Self-Administration Influences Drug Seeking in the Rat: Optogenetic Evidence for a Role of the Prelimbic Cortex
}

\author{
Elena Martín-García ${ }^{1,2,3}$, Julien Courtin ${ }^{2,4}$, Prisca Renault ${ }^{1,2,8}$, Jean- François Fiancette ${ }^{1,2,8}$, Hélène Wurtz ${ }^{2,4}$, \\ Amélie Simonnet ${ }^{2,4}$, Florian Levet ${ }^{5,6,7}$, Cyril Herry ${ }^{2,4,9}$ and Véronique Deroche-Gamonet*, ${ }^{*, 2,9}$ \\ 'INSERM U862, Pathophysiology of Addiction, NeuroCentre Magendie, 146 rue Léo Saignat, Bordeaux, Cedex, France; ${ }^{2}$ University of Bordeaux, \\ 146 Rue Léo Saignat, Bordeaux, Cedex, France; ${ }^{3}$ Departament de Ciencies Experimentals i de la Salut, Universitat Pompeu Fabra, PRBB, CI Dr \\ Aiguader 88, Barcelona, Spain; ${ }^{4}$ NSSERM U862, Neuronal Circuits of Associative Learning, NeuroCentre Magendie, 146 rue Léo Saignat, \\ Bordeaux, Cedex, France; ${ }^{5}$ University of Bordeaux, Bordeaux Imaging Center, Bordeaux, France; ${ }^{6}$ CNRS, Bordeaux Imaging Center, Bordeaux, \\ France; ' INSERM, Bordeaux Imaging Center, Bordeaux, France
}

\begin{abstract}
High-frequency intake and high drug-induced seeking are associated with cocaine addiction in both human and animals. However, their relationships and neurobiological underpinnings remain hypothetical. The medial prefrontal cortex (mPFC), basolateral amygdala (BLA), and nucleus accumbens (NAc) have been shown to have a role in cocaine seeking. However, their involvement in regulating highfrequency intake and high cocaine-induced seeking is unclear. We manipulated frequency of cocaine self-administration and investigated whether it influenced cocaine seeking. The contribution of the aforementioned structures was evaluated using changes in expression of the immediate early gene c-Fos and targeted optogenetic manipulations. Rats that self-administered at High frequency (short inter-infusion intervals allowed by short time-out) showed higher cocaine-induced seeking than low frequency rats (long inter-infusions intervals imposed by long time-out), as measured with cocaine-induced reinstatement. c-Fos was enhanced in High frequency rats in the prelimbic $(\mathrm{PL})$ and infralimbic $(\mathrm{IL})$ areas of the $\mathrm{MPFC}$, the BLA, and the NAc core and shell. Correlational analysis of c-Fos revealed that the PL was a critical node strongly correlated with both the IL and NAc core in High frequency rats. Targeted optogenetic inactivation of the PL decreased cocaine-induced reinstatement, but increased cocaine self-administration, in High frequency rats. In contrast, optogenetic activation of the PL had no effect on Low frequency rats. Thus, high-frequency intake promotes a PL-dependent control of cocaine seeking, with the PL exerting a facilitatory or inhibitory effect, depending on operant contingencies. Individual differences in cocaineinduced PL activation might be a source of vulnerability for poorly controlled cocaine-induced seeking and/or cocaine intake. Neuropsychopharmacology (20I4) 39, 23 I7-2330; doi:I0.I038/npp.20 I4.66; published online I6 April 2014
\end{abstract}

\section{INTRODUCTION}

Cocaine addiction is typically associated with the emergence of frequent (bingeing) use (Dackis and O'Brien, 2001; Gawin and Kleber, 1988; Gawin, 1991) and high cocaine-induced seeking behavior (Gawin and Khalsa-Denison, 1996; Jaffe et al, 1989). Using a multi-symptomatic model of cocaine addiction in the rat (Deroche-Gamonet and Piazza, 2014; Deroche-Gamonet et al, 2004), we revealed that highfrequency self-administration (short Inter-Infusion Intervals) and high cocaine-induced seeking, as measured in a

\footnotetext{
*Correspondence: Dr V Deroche-Gamonet, CRI U862, Team Pathophysiology of Addiction-Group Psychobiology of Addiction, Neurocentre Magendie, I 46 rue Léo Saignat, Bordeaux, Cedex 33077, France, Tel: +335575736 80, Fax: +335575736 69,

E-mail: veronique.deroche@inserm.fr

${ }^{8}$ These authors contributed equally to this work.

${ }^{9}$ These authors contributed equally to this work.

Received 6 December 2013; revised 9 March 2014; accepted 9 March 2014; accepted article preview online 17 March 2014
}

reinstatement test, are concomitant with the expression of an addiction-like behavior. Also, they precede and predict its development, suggesting a possible role in the addiction process (Belin et al, 2009). However, the relationship between high-frequency intake and increased sensitivity to the incentive effects of cocaine, and their possible role in compulsive drug use, as well as the neuronal structures mediating these behavioral effects, remain unknown.

In the present context, the medial prefrontal cortex, in particular the prelimbic area (PL), is a target of interest. The $\mathrm{PL}$ is involved in regulating emotional and cognitive aspects of goal-directed behaviors (Vertes, 2006). Evidence implicates it in promoting drug seeking. Indeed, as a part of a network including the infralimbic cortex (IL), the basolateral nucleus of the amygdala (BLA) and the nucleus accumbens core and shell (NAc core, NAc shell), the prelimbic cortex (PL) has been shown to have a key role in promoting drug seeking in reinstatement procedures (Di Ciano and Everitt, 2004; Kalivas and McFarland, 2003; LaLumiere et al, 2012; Peters et al, 2008; Stefanik et al, 2013). For instance, PL, but 
not IL, inactivation reduces drug-, stress-, CS- and contextinduced reinstatement of cocaine seeking (Ball and Slane, 2012; Bossert et al, 2013; Capriles et al, 2003; McLaughlin and See, 2003). Moreover, this effect seems to be pathway specific, because the optical inhibition of PL neurons projecting to the NAc core blocked cocaine-induced reinstatement (Stefanik et al, 2013).

However, the contribution of the PL could be more complex than solely promoting drug seeking after extinction. Paradoxically, data in both rat models and humans also implicate the PL in inhibition of drug seeking. After extinction, pharmacological inactivation of the PL, did not reduce but, increased nose poking for alcohol, when behavior was reinforced by the drug (Willcocks and McNally, 2013). PL would also contribute to foot-shockinduced suppression of cocaine self-administration in rats. Indeed, optical activation of the PL inhibited responses in rats showing resistance to punishment (Chen et al, 2013). Inversely, optical inactivation of the PL promoted cocaine seeking in rats responsive to punishment by foot-shocks. Also, rat's ability to inhibit cocaine-induced reinstatement of drug seeking, when presented with a discriminative cue signaling drug unavailability, depends on neuronal activation within the PL (Mihindou et al, 2013). In the same line, neuroimaging studies revealed that drug addicts can suppress drug craving by recruiting the lateral prefrontal cortex (for review (Goldstein and Volkow, 2011)), considered as the primate homolog of the rat PL (Vertes, 2006).

Altogether these observations question the nature of the control of cocaine seeking by the PL. Therefore, it is also unclear whether and how the PL is involved in the regulation of high cocaine-induced reinstatement of drug seeking associated with high-frequency cocaine intake.

In the present work, we first manipulated frequency of cocaine self-administration in rats and investigated whether it altered cocaine-induced reinstatement of drug seeking. Next, using c-Fos expression as a marker of neuronal activity, we tested the impact of High vs Low frequency cocaine self-administration on neuronal activity in the PL and aforementioned network including IL, Nac core, Nac shell and BLA. Finally, applying targeted optogenetic manipulations, we aimed at disentangling the role of the PL in cocaine seeking, in both cocaine-induced reinstatement and in a regular self-administration session, as a function of frequency of cocaine intake.

\section{MATERIALS AND METHODS}

\section{Animals}

Male Sprague-Dawley rats $(n=25$ for Experiment 1 and $n=33$ for Experiment 2) weighing 280-300 g at the beginning of the experiments were single housed under a $12 \mathrm{~h}$ reverse dark/light cycle (on 2000 hours, off 0800 hours). Details are provided in the Supplementary Information.

\section{Adeno-Associated Viral Vectors}

Concentrated Adeno-Associated Viral vectors (AAV) encoding channelrhodopsin-2 (AAV-CamKIIa-hChR2(H134R)-EYFP, serotype 5, Vector Core, University of North Carolina)
(ChR2 group), archaeorhodopsin (AAV-CamKII-ArchTEYFP, serotype 5, Vector Core, University of North Carolina) (ArchT group), or enhanced yellow fluorescent protein (EYFP) (AAV-CamKIIa-EYFP, serotype 5, Vector Core, University of North Carolina) (Control, EYFP group), under the control of the calcium calmodulin kinase II (CamKII) promoter were used. Under optical stimulation, excitatory projection neurons expressing ArchT and ChR2 were inhibited and activated, respectively. The EYFP group was used as a control for psychopharmacological consequences of AAV injection and optical stimulation. Following the completion of the self-administration experiment, brain immunohistochemistry was performed on all rats to localize virus expression, through EYFP expression, and to control for optic fiber placement.

\section{Drugs}

Cocaine hydrochloride was obtained from COOPER (Bordeaux, France) and dissolved in sterile 0.9\% physiological saline. Ketamine hydrochloride $(80 \mathrm{mg} / \mathrm{kg}$ ) (Imalgène 1000; Rhône Mérieux, Lyon, France) and xylazine hydrochloride (16 mg/kg) (Rompun; Rhône Mérieux, Lyon, France) were mixed with saline and administered intraperitoneally in a volume of $20 \mathrm{ml} / \mathrm{kg}$ of body weight.

\section{Surgeries (Detailed in the $S I$ )}

Surgery for self-administering rats (Experiment 1). A silastic catheter was implanted in the right jugular vein, as previously described (Belin et al, 2009).

Surgeries for brain optogenetic manipulations in selfadministering rats (Experiment 2). Rats underwent two surgeries 5 days apart. First, AAV expressing $\operatorname{ChR} 2(n=11)$, $\operatorname{ArchT}(n=15)$, or EYFP (controls, $n=7)$ (approximately $0.4 \mu \mathrm{l}$ per hemisphere) were bilaterally injected using glass pipettes (tip diameter $10-20 \mu \mathrm{m}$ ) connected to a picospritzer (Parker Hannifin Corporation) into the PL, at the following coordinates: $3.2 \mathrm{~mm}$ anterior to the bregma, $0.5 \mathrm{~mm}$ lateral of midline, $2.6 \mathrm{~mm}$ below the cortical surface, according to a classical stereotaxic procedure based on the rat brain atlas (Paxinos and Watson, 2005). Second, rats were implanted with an intravenous catheter in the jugular vein and bilateral optic fibers directed to the PL (diameter: $200 \mu \mathrm{m}$; numerical aperture: 0.37; flat tip, Doric Lenses). Implants were fixed to the skull with Super-Bond dental cement (Sun Medical, Shiga, Japan).

\section{Intravenous Self-Administration Apparatus}

The self-administration setup, consisting of 40 self-administration chambers made of plexiglas and metal (Imetronic, Pessac, France), and equipped with holes as operant manipulanda (Belin et al, 2009), is detailed in the Supplementary Information.

Cocaine self-administration. Cocaine self-administration $(0.8 \mathrm{mg} / \mathrm{kg} / 40 \mu \mathrm{l}$ infusion, i.v.) sessions were conducted daily, between 1000 and 1300 hours, 5-7 days per week. A blue cue light served as a discriminative stimulus signaling drug availability and was off during time-out (TO). 
Introduction of the rat's nose into one hole (active device) turned on the white cue light, located above it, and, then $1 \mathrm{~s}$ later, the infusion pump was switched on for $2 \mathrm{~s}$. The white cue light remained on for a total of $4 \mathrm{~s}$. Nose-pokes in the other hole (inactive device) had no scheduled consequences.

Cocaine-induced reinstatement. During cocaine-induced reinstatement tests, cocaine was not available contingently. Following a 90 -min (Experiment 1) or 50-min (Experiment 2) period of extinction, four increasing doses of cocaine $(0.2,0.4,0.8$, and $1.6 \mathrm{mg} / \mathrm{kg} /$ infusion, i.v. $)$ were delivered non-contingently at a rate of one infusion every $30 \mathrm{~min}$. Over the whole session, active and inactive nose-pokes were recorded but were without scheduled consequences (details in the Supplementary Information). The discrete cocaineassociated cue light was not delivered during reinstatement sessions, either contingently or non-contingently.

In total, in Experiments 1 and 2, rats were tested in selfadministration (and reinstatement) sessions over 35 and 39 days, respectively.

\section{c-Fos Immunohistochemistry}

Brain sampling and staining. Rats were anesthetized by inhalation of $2.5 \%$ isoflurane using oxygen as a vehicle and perfused transcardially with a solution of $9 \mathrm{~g} / \mathrm{l} \mathrm{NaCl}$ followed by $4 \%$ of paraformaldehyde in phosphate buffer $(\mathrm{PB} ; \mathrm{pH}=7.4)$. Four drug-naive control rats were killed at the same time. They were moved daily to the self-administration room together with the other rats. They were manipulated as the others, spent the session time in the selfadministration experimental room, but in their home cage. The tissue for immunohistochemistry was processed as previously described (Herry and Mons, 2004) (details in the Supplementary Information).

Visualization and quantification of c-Fos expression. The stained sections of the brains were visualized at $\times 10$ magnification using an epifluorescent microscope coupled to a high-sensitivity camera driven by the MetaMorph software. Transmission acquisition mode was used to scan slides. Slide area was reconstructed with mosaic images produced with a motorized stage Scan. Equipment is detailed in the Supplementary Information.

Using Image software, c-Fos-positive cells were quantified in the five structures of interest in both hemispheres and expressed as mean number per $\mathrm{mm}^{2}$ of standardized fixed-area shapes (coordinates according to the rat brain atlas (Paxinos and Watson, 2005); coordinates relative to the bregma): IL $(3.24 \mathrm{~mm})$, PL $(3.24 \mathrm{~mm})$, Nac core $(1.20 \mathrm{~mm})$, Nac shell $(1.20 \mathrm{~mm})$, BLA $(-2.64 \mathrm{~mm})$. Areas sampled are indicated by black squares in Figure 4 and Supplementary Figure S5. For each region, the results of two successive slices were averaged. At all stages, the experimenter was blind to the experimental groups. For details see Supplementary Information.

\section{Optogenetic Setup and Stimulation Protocol}

Optogenetic manipulations were performed 38-41 days after virus injection. Stimulation of ChR2 or ArchT (approximately $5 \mathrm{~mW}$ per implanted fiber, $250 \mathrm{~ms}$ pulse width, $1 \mathrm{~Hz}$, during the entire session) was delivered using a TTL pulse generator.

Each implanted optic fiber was connected to a LED module $(465 \mathrm{~nm})$ mounted on a dual LED Commutator, which was connected to a LED driver system (PlexBright, Plexon, USA) powered by the TTL pulse generator (Imetronic, Pessac, France). A dual pharmacology/optic fiber-motorized commutator (Imetronic, Pessac, France) assured smooth and combined rotation of optical fibers and cocaine self-infusion line, allowing animals to move as freely as usual. Details are provided in the Supplementary Information.

Efficiency of optical stimulation in activating/inhibiting neuronal activity in the PL was controlled in independent groups of rats injected with $\operatorname{ChR2}(n=2)$ and $\operatorname{ArchT}(n=2)$, using in vivo single unit electrophysiological recordings in behaving rats (Supplementary Information for details and Supplementary Figure S7).

\section{EYFP Immunohistochemistry}

Brain sampling and visualization of EYFP expression. Brain sampling was similar as for c-Fos immunohistochemistry. After short post-fixation, $80-\mu \mathrm{m}$-thick coronal sections were cut and mounted using Vectashield (Vector Laboratories, Peterborough, UK). Images were acquired using a $\times 20$ digital slide scanner Nanozoomer 2.0 (Hamamatsu, Massy, France).

\section{Calculation of Brain Cocaine Concentrations}

In Experiment 1, whole-brain levels of cocaine were mathematically evaluated using a two-compartment model for rats receiving a chronic cocaine i.v. regimen (Zimmer et al, 2011; 2012) (details in the Supplementary Information).

\section{Experimental Procedures}

Acquisition of cocaine self-administration (Experiments 1 and 2). Three to five days after surgery (the second surgery in case of Experiment 2), rats were trained to selfadminister cocaine $(0.8 \mathrm{mg} / \mathrm{kg} /$ infusion $)$ on an FR3, then FR5, (3 and 5 days, respectively) schedule of reinforcement, with a 40-s TO. The session ended after $180 \mathrm{~min}$ or 30 infusions. Criteria for acquisition of cocaine self-administration were defined by a stable number of self-infusions $( \pm 10 \%)$ and significant discrimination between active and inactive holes, over at least three consecutive selfadministration sessions.

Experiment 1: implementation of procedures promoting High vs Low frequency cocaine self-administration: effect on cocaine-induced reinstatement and cocaine-induced c-Fos expression. After the eight acquisition sessions described above, three homogeneous groups were formed ( $n=8-9$ per group) and assigned to $2-(2 \min T O)$, 4- $(4 \operatorname{minTO})$, or 6 -min $(6 \operatorname{minTO})$ TO conditions. The procedure was similar to the one applied during the last five acquisition sessions (FR5), except for the TO and a reduction of the maximal number of infusions to 25 . The number of infusions was reduced to minimize possible differences between the three groups (notably allowing the 


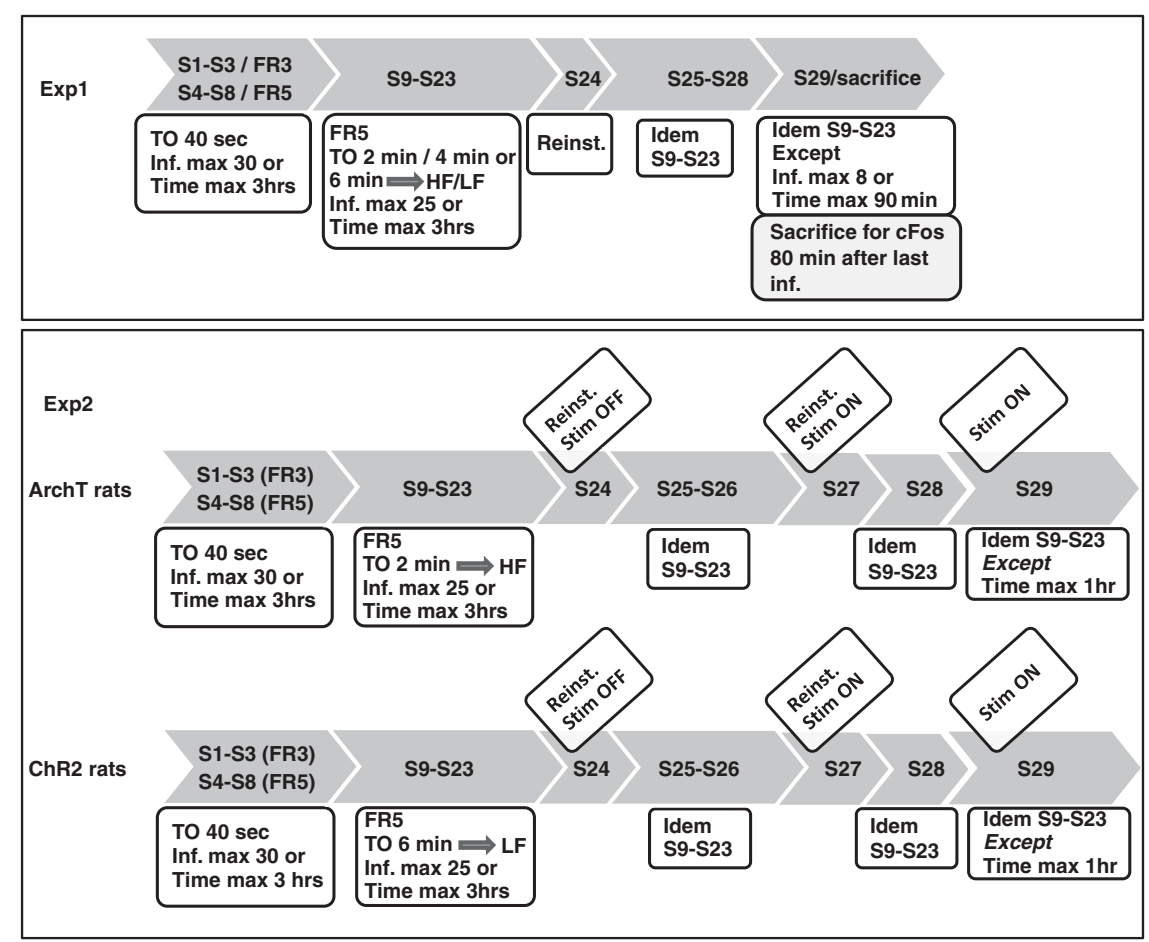

Figure I Experimental procedures applied in Experiments I (Exp I) and 2 (Exp2). FR, fixed ratio; S, session; and TO, Time-Out. Stim refers to optical stimulation. ArchT and ChR2 refer to the two groups of rats administered with AAV-CamKIl-ArchT-EYFP and AAV-CamKIla-hChR2(HI34R)-EYFP in the PL for optical inactivation and activation, respectively. High frequency (HF) and Low frequency (LF) protocols were applied to ArChT and ChR2 rats, respectively. Control rats received EYFP in the PL and were tested for self-administration (HF) as ArchT rats.

6 minTO group performing the maximal number of infusions within the allocated time). This procedure generated different frequencies of cocaine self-administration evaluated by the mean inter-infusions interval (III) and allowed separation in two groups showing short (High frequency rats, $n=12$ ) and long III (Low frequency rats, $n=13$ ) (see Results section). To evaluate the incentive properties of the drug, cocaine-induced reinstatement (Belin et al, 2009; Epstein et al, 2006) was tested on session 24, ie, after 15 sessions of this new TO procedure.

After cocaine-induced reinstatement, four additional training sessions were performed in the same conditions as sessions 9 to 23. Then a subset of 12 representative rats (High frequency, $n=6$; Low frequency, $n=6$ ) were submitted to a last session using a similar procedure, except that session stopped after eight infusions or $90 \mathrm{~min}$. Following the session, rats were immediately returned to the vivarium and killed $80 \mathrm{~min}$ later. c-Fos expression reflects strong neuronal activity about $1 \mathrm{~h}$ before sacrifice. The 80-min lapse was chosen because it allowed cocaine to decrease to comparable low brain levels in the two groups about $1 \mathrm{~h}$ before sacrifice (see schematic procedures in Figure 1).

Experiment 2: optogenetic manipulation of neuronal activity in the PL: effect on cocaine-induced reinstatement and cocaine self-administration in High and Low frequency rats. On the basis of Experiment 1 , we observed a higher cocaine-induced reinstatement in High frequency than in Low frequency rats. As the PL had been shown to promote drug seeking in reinstatement procedures, we hypothesized that the difference between the two groups resulted from a hyper- $v s$ a hypoactivity of the PL in High vs Low frequency rats. Therefore, we planned to inactivate the PL in the High frequency and activate it in the Low frequency rats.

After acquisition, EYFP $(n=7)$ and $\operatorname{ArchT}(n=15)$ rats were assigned to the 2 minTO procedure (High frequency), and $\operatorname{ChR} 2$ rats $(n=11)$ were assigned to the $6 \min T O$ procedure (Low frequency). Accordingly, the $2 \operatorname{minTO}$ generated a higher frequency of intake compared with the $6 \min T O$. Each rat was tested once between sessions 16 and 19, with optical fibers connected, to identify possible disruptive effects of head connection on self-administration behavior. Two tests of cocaine-induced reinstatement were performed, on sessions 24 and 27; optical stimulation being applied in session 27. On sessions 25, 26, and 28, a basal FR5 procedure was applied, with a $2 \operatorname{minTO}$ or $6 \operatorname{minTO}$ depending on the group. On session 29, rats were tested in a 1 - $\mathrm{h}$ cocaine self-administration session with the same basal FR5 procedure and with optical stimulation applied.

After experiment completion, eight rats were excluded (one EYFP, four ArchT, and three ChR2) for reasons described in the Supplementary Information.

\section{Data Analysis}

Cocaine self-administration. Total responses in the active and inactive holes and total number of cocaine infusions per self-administration session were considered. 
Frequency of cocaine intake. Frequency of cocaine selfadministration was determined according to III. It was calculated as the mean time, in minutes, between each infusion and the following one.

Cocaine-induced reinstatement. For each hole, responses were segregated in 5-min blocks. Extinction behavior was analyzed by comparing responses in the active $v s$ inactive holes over the first $90 \mathrm{~min}$ (Experiment 1) or $50 \mathrm{~min}$ (Experiment 2) of the session. Cocaine-induced reinstatement (the last $120 \mathrm{~min}$ of the session), as a function of the dose, was analyzed by comparing responses in the active and inactive holes over the first $15 \mathrm{~min}$ following each cocaine infusion. For Experiment 1, the time course of active responses over the $120 \mathrm{~min}$ following the first cocaine infusion is also presented.

c-Fos immunohistochemistry. Beside quantitative differences in the mean number of c-Fos-positive cells per $\mathrm{mm}^{2}$, indices of altered functional connectivity between the five structures of interest were also investigated. First, a descriptive approach using Hierarchical Ascendant Cluster Analysis was applied. It provides a qualitative picture (translated into a cluster tree) of the hierarchical distance between structures. Second, to support and complete the descriptive cluster analysis, a correlation approach was applied. It allows evaluating, and statistically testing, the nature and strength of the relationships between c-Fos activities in pairs of brain structures. See Supplementary Information for details.

Effect of optogenetic stimulation on cocaine-induced reinstatement and cocaine self-administration behavior. As eight rats were excluded, analyses were performed on 25 rats (six EYFP, eleven ArchT, and eight ChR2) using within-subject designs. For cocaine-induced reinstatement, the non-stimulated condition (session 24) was compared with the stimulated condition (session 27). Similarly, for cocaine self-administration, we compared behavior in session 29 ( $1 \mathrm{~h}$ session during which optical stimulation was applied) with behavior expressed over the same period of time during basal self-administration sessions (mean over sessions 26 and 28). Active and inactive nose-pokes, infusions, and III were analyzed.

\section{Statistical Analysis}

Self-administration behavior was analyzed using repeatedmeasures ANOVA with time (number of sessions, 1 or $5 \mathrm{~min}$ time blocks) or hole (active $v s$ inactive) or stimulation (On $v s$ Off) as a within-subject factor and group (High vs Low frequency/2minTO vs 4 minTO vs 6minTO/EYFP vs ChR2 vs ArchT) as a between-subject factor. c-Fos expression data were analyzed using one-way ANOVA with experimental groups as a between-subject factor. For both self-administration and c-Fos data, significant main effects or interactions were explored by pairwise comparisons of means using the Newman Keuls post hoc test. Pearson's correlation analyses were used to investigate correlations in c-Fos expression data between the five structures of interest. Student's $t$-test was used to investigate effects of optical stimulation on firing frequency of PL neurons in the electrophysiological experiment. The results are presented as mean \pm SEM. Differences were considered significant at $p<0.05$. The statistical analyses were performed using the STATISTICA 6.0 data analysis software system (StatSoft, Tulsa, OK, USA, 2001).

\section{RESULTS}

\section{Acquisition of Cocaine Intravenous Self-Administration and Induction of Distinct Frequencies of Intake}

Experiment 1. Over the first eight sessions, rats acquired cocaine self-administration (FR3/FR5, $40 \mathrm{~s}$ TO). On the basis of the last three acquisition sessions, three homogeneous groups were formed. Evidence of self-administration acquisition (Supplementary Figure S1) and homogeneity of experimental groups (Supplementary Figure S2) is described in the Supplementary Information.

Each group was then given a distinct TO period $(2,4$, or $6 \mathrm{~min})$. The procedure immediately produced different frequencies of intake as measured by the mean III (Supplementary Figure S3A-C). In each group, the IIIs were normally or log-normally distributed and centered on 3, 5, and $7 \mathrm{~min}$, respectively. Therefore, low and high extremes in the $4 \min T O$ group overlapped with the 2 minTO and 6 minTO groups, respectively. Consequently, all rats were pooled and divided in two groups based on the median III value: High III $(n=13$, mean III $=6.20 \pm 0.25$, for Low frequency rats) and Low III $(n=12$, mean III $=3.49 \pm 0.22$, for High frequency rats). These two groups did not differ for III over the eight acquisition sessions, but did over the 19 following sessions, as expected (Figure 2a). Notably, the two groups were not significantly different for nose-poke responses and discrimination (Figure 2c) or for cocaine intake during all 27 self-administration sessions (Figure 2b).

Experiment 2. All groups acquired cocaine self-administration similarly. Starting session 9, ArchT and EYFP rats, trained with the 2 minTO condition, constituted High frequency rats. The $C h R 2$ group, trained with the 6 minTO condition, constituted Low frequency rats.

\section{Cocaine-Induced Reinstatement is Increased in High vs Low frequency Rats (Experiments 1 and 2)}

In both Experiments 1 and 2, rats were tested for cocaineinduced reinstatement in session 24. In Experiment 2, rats equipped for PL optogenetic stimulation were tested in the control condition; ie, the optic fibers were connected but the stimulation was not activated. EYFP and ArchT rats, trained with the $2 \operatorname{minTO}$ condition, did not differ and were pooled as High frequency rats.

Extinction. In Experiment 1, High frequency rats nosepoked more than Low frequency rats (Group effect, $\mathrm{F}(1,23)=4.86, p<0.05)$. This effect relied on more nose-pokes in the active hole during the first two 5-min time-blocks $\left(\right.$ Group $\times$ Hole $\times$ Time, $\left.\mathrm{F}_{(17,391)}=2.06, p<0.001\right)$. Importantly, however, the two groups showed a similar extinction rate, 

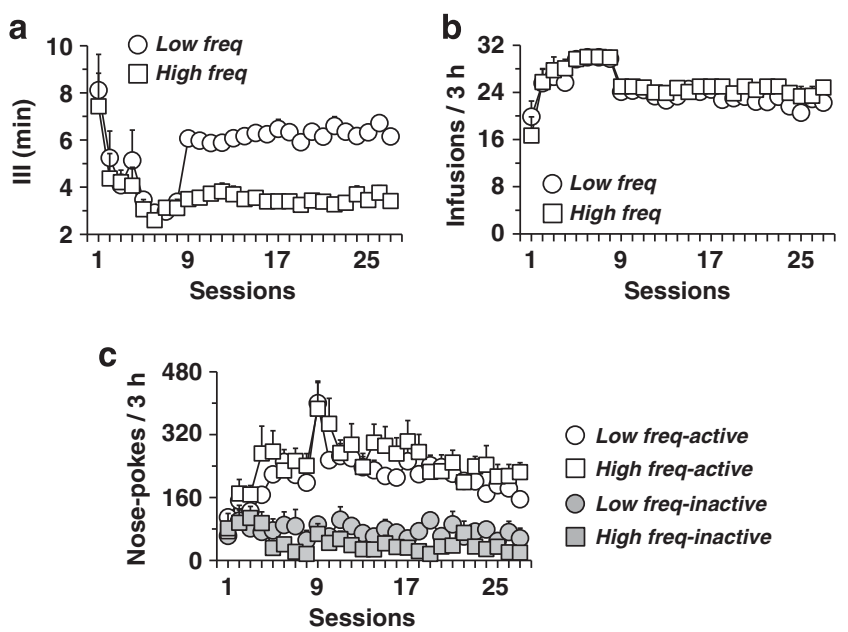

Figure 2 Cocaine self-administration in High (High freq) and Low frequency (Low freq) rats. The 8 acquisition sessions and 19 sessions of the distinct time-out (TO) procedure are shown. Starting session 9, distinct TOs were introduced that produced high and low frequencies of intake. (a) Daily mean inter-infusion interval (III) differed significantly between High and Low frequency rats, starting session 9, (b) intake of cocaine, as measured by the number of infusions per session, as well as mean active and inactive nose-pokes per session (c), were similar in the two groups. Data are expressed as mean \pm SEM ( $n=12$ to 13 per group).

as the discrimination time courses were similar over the first $15 \mathrm{~min}$, ie, until discrimination was lost (Figure 3a). At the end of the 90-min extinction period, before noncontingent cocaine infusions, the two groups showed similar low levels of responding in both active and inactive holes.

Notably, in Experiment 2, High and Low frequency rats did not differ in extinction and the number of nose-pokes was lower than that in Experiment 1 (data not shown). We hypothesize that dummy connection of optical fibers was responsible for this decrease in operant responses, when cocaine was not available. Indeed decrease in responses (and self-infusions) was not observed when rats were head-connected during a regular self-administration session (tested between sessions 16 and 19 for habituation, data not shown). Importantly, however, the time course of extinction was similar in the two groups and at the end of the $50 \mathrm{~min}$ of the extinction period, before non-contingent cocaine infusions, the two groups showed similar low levels of responding in both active and inactive holes.

Reinstatement. On session 24, in both experiments, noncontingent cocaine infusions produced reinstatement of extinguished instrumental responses, inducing a specific and dose-dependent increase of active responses (Figure 3cExperiment 1-Figure 3d-Experiment 2). In both cases also, High frequency rats responded more than Low frequency rats, specifically in the active hole (Group $\times$ Hole, $\mathrm{F}_{(1,23)}=$ $6.52, p<0.05 ; \mathrm{F}_{(1,22)}=1.69, p<0.05$, respectively). A time course of responses over the $120 \mathrm{~min}$ following the first non-contingent cocaine infusion is displayed in Figure $3 \mathrm{~b}$ (Experiment 1).
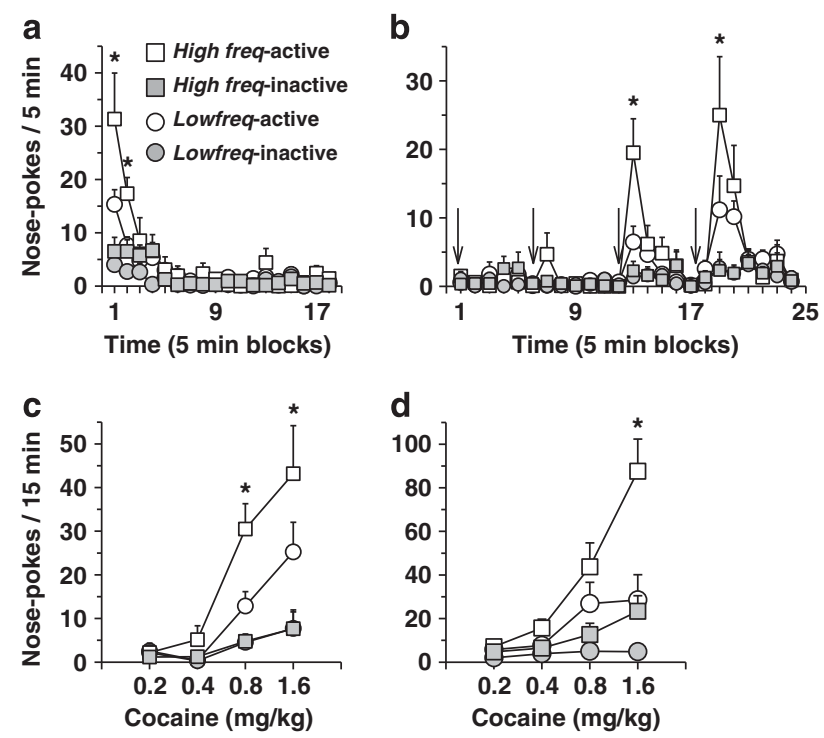

Figure 3 Reinstatement of cocaine self-administration behavior in High (High freq) and Low frequency rats (Low freq). The session lasted 210 min (90 min extinction +120 min of cocaine-induced reinstatement). (a-c) Experiment I. (a) Time course of active and inactive nose-pokes during the $90 \mathrm{~min}$ period of extinction. Active nose-pokes were higher in the High frequency rats, over the first $10 \mathrm{~min}$. Behavior was fully extinguished in both groups when the first cocaine dose was infused. (b) Time course of active nose-pokes following four non-contingent infusions (one every $30 \mathrm{~min}$ ) of increasing doses of cocaine (time of infusions is indicated by a black arrow). Cocaine induced a dose-dependent increase of active response, an effect that was higher in High frequency rats. (c) Dose-response of reinstatement. Total active and inactive responses over the first $15 \mathrm{~min}$ following each cocaine infusion are represented ( $n=12$ to 13 per group). ${ }^{*} p<0.05$ as compared with Low freq rats. (d) Experiment 2: total active and inactive responses over the first fifteen minutes following each cocaine infusion are represented. Rats had been administered with virus and optical fibers into the PL before self-administration training. As for Experiment I, High frequency rats showed higher cocaine-induced reinstatement than Low frequency rats $(n=17$ High freq (EYFP \& ArchT) and $n=11$ Low freq $(C h R 2))$. $p<0.05$ as compared with Low freq rats. Data are expressed as mean \pm SEM

\section{Cocaine Self-Administration Induces Distinct c-Fos Expression Profiles in High vs Low frequency Rats}

Last self-administration session before sacrifice. In Experiment 1, twelve representative rats (six High and six Low frequency) were tested in a last self-administration session that ended after eight self-administered infusions or if $90 \mathrm{~min}$ had elapsed (mean III: $3.68 \pm 0.32$ and $6.72 \min \pm 0.18$, respectively, over the 19 self-administration sessions of the specific TO procedure-Group effect, $\left.\mathrm{F}_{(1,10)}=66.41, p<0.001\right)$. All rats completed the eight infusions within the allocated time; the longest session lasted $80 \mathrm{~min}$. Each rat was killed $80 \mathrm{~min}$ after completion of its last infusion.

During this last session, the difference in III was maintained, though slightly amplified (Group effect, $\mathrm{F}_{(1,10)}=$ 16.47, $p<0.005$; low: $8.66 \mathrm{~min} \pm 0.76$, high: $3.92 \pm 0.88$ ). Accordingly, the mean duration of the session was significantly different between the two groups (Group effect, $\mathrm{F}_{(1,10)}=14.11, p<0.01$; low: $59.33 \mathrm{~min} \pm 10$; high: $29.16 \mathrm{~min} \pm 7.55)$. The self-administration behavior and 


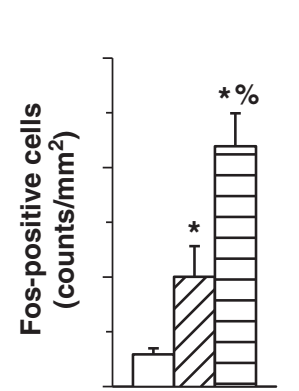

PL
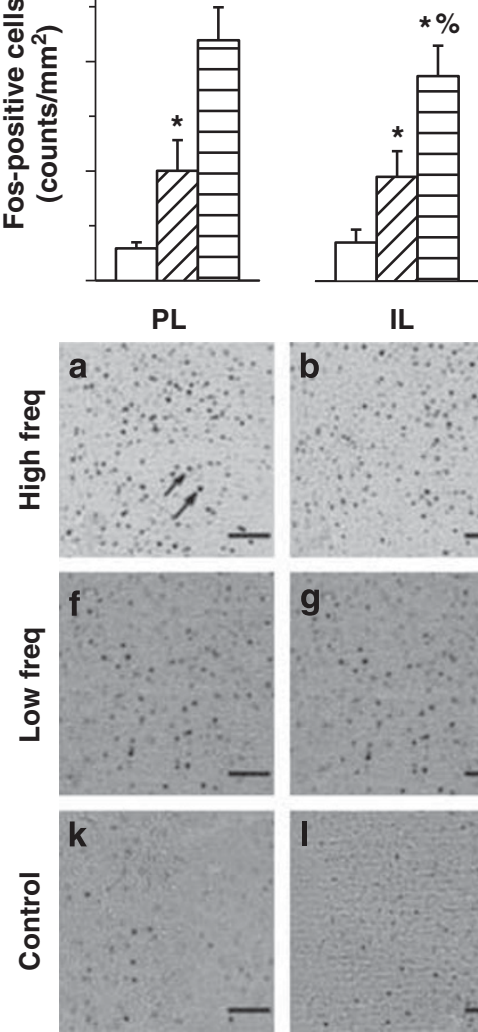

IL
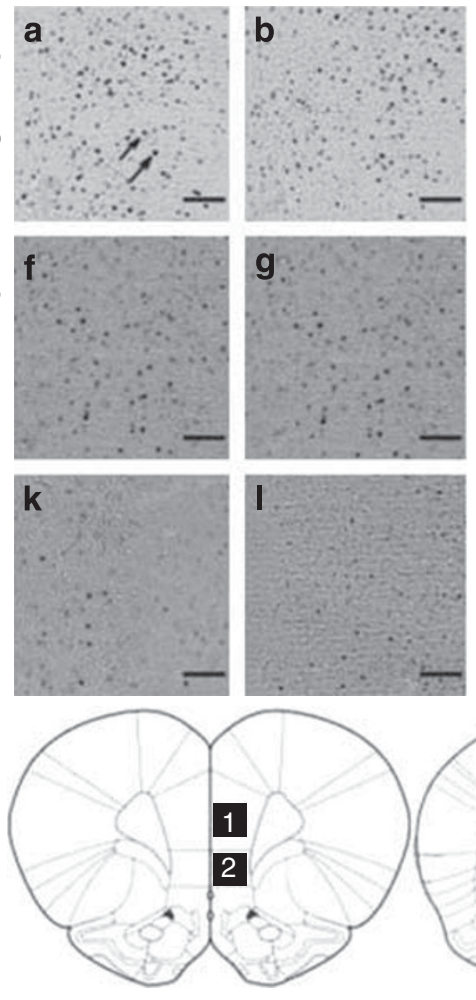

Bregma +3.24 mm
Control

$Z 7$ Low freq

$\equiv$ High freq

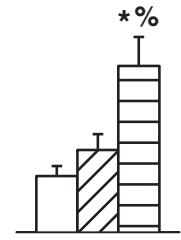

Nac core
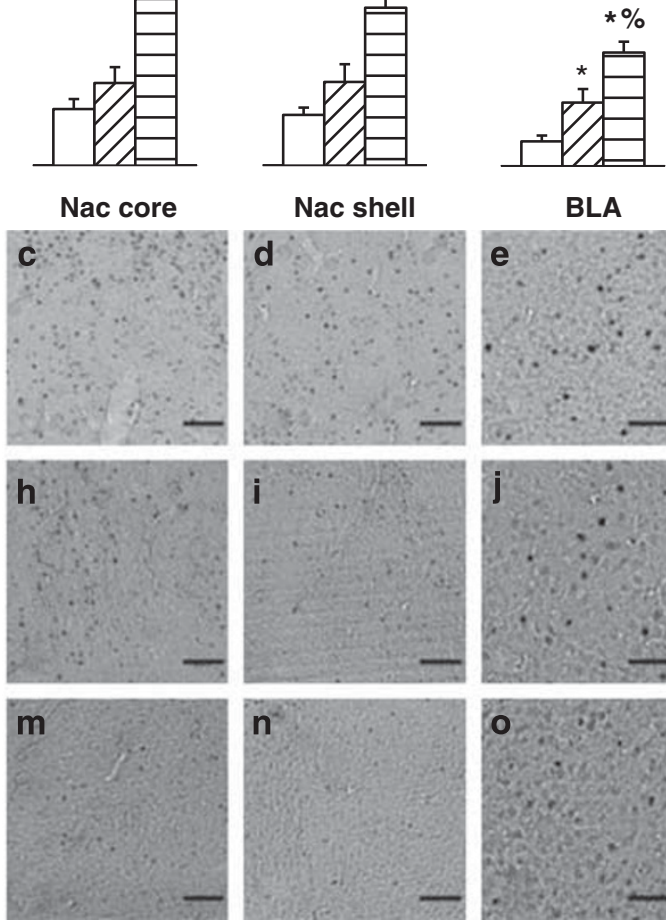

BLA
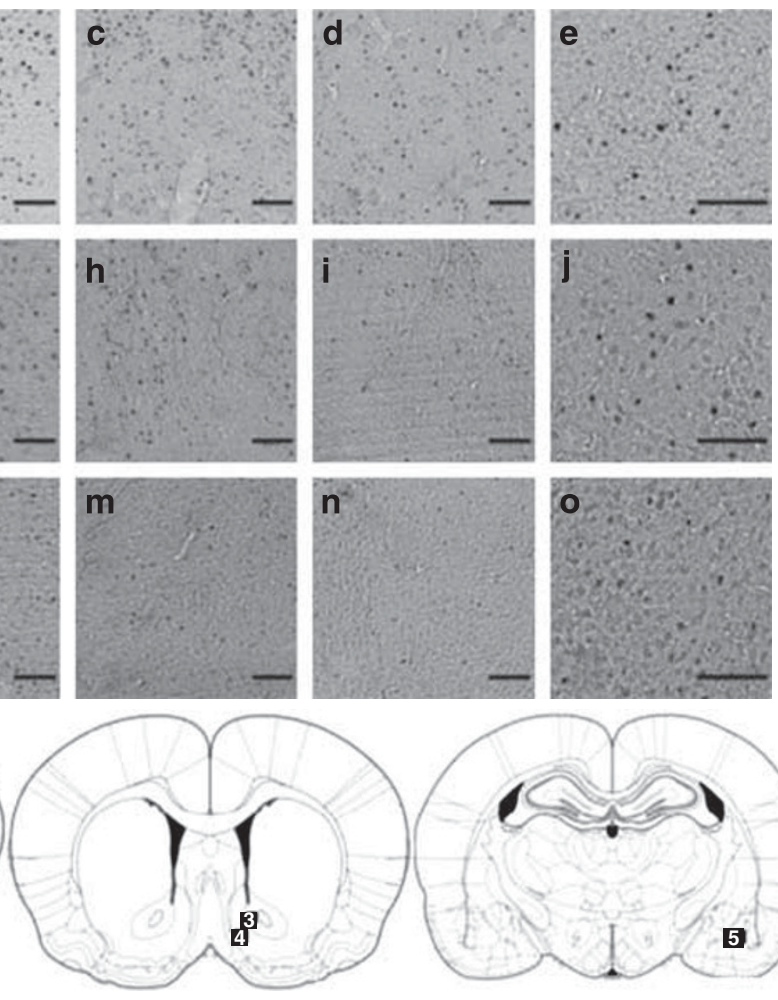

Bregma $+1.20 \mathrm{~mm}$

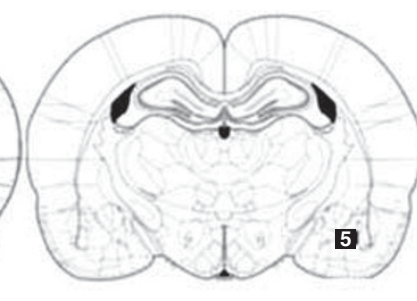

Bregma -2.64 mm

Figure 4 In the upper part, mean number of c-Fos-positive cells/mm² in PL, IL, NAc Core, NAc Shell, and BLA in High (High freq) and Low frequency (Low freq) rats. The three groups significantly differed for c-Fos expression in the five structures of interest. In the prelimbic cortex (PL), infralimbic cortex, (IL) and basolateral amygdala (BLA), cocaine self-administration increased c-Fos expression, but High frequency rats showed higher c-Fos expression than Low frequency rats. In the nucleus accumbens core (NAc core) and nucleus accumbens shell (NAc shell), High frequency was specifically associated with increased c-Fos expression, as Low frequency rats did not differ from Control rats. Data are expressed as mean \pm SEM ( $n=4-6$ per group). *: significant as compared with Control, \%: significant as compared with Low frequency rats. In the medium part, representative pictures of c-Fos staining in the five structures of interest for the three experimental groups (a-e: High frequency, f-j: Low frequency, and k-O: Control). In the bottom part, pictures are schematic representations of coronal sections of the rat brain taken at 3.24, I.20, and $-2.64 \mathrm{~mm}$ from the bregma (Paxinos and Watson, 2005). Numbers in the sections represent the regions analyzed for c-Fos: (I) PL; (2) IL; (3) NAc core; (4) NAc shell; (5) BLA. All images were taken at $\times 10$ magnification. Scale bar is equal to I00 $\mu$ m. Visible c-Fos protein expression was manifested as dark ovals (highlighted by arrows).

cocaine-induced reinstatement of these two representative groups are portrayed in Supplementary Figure S4.

Quantitative differences in c-Fos expression levels between Low and High frequency rats. The three groups (High frequency, Low frequency, and cocaine-naive animals) significantly differed in c-Fos expression in the five structures of interest (Figure 4) (Group effect, $\mathrm{F}_{(2,13)}=16,73$, $p<0.0005)$.
C-Fos expression was higher in the NAc core and NAc shell of the High frequency group. Cocaine-naive (control) and Low frequency rats were similar and significantly lower than the High frequency. For the other three structures (PL, IL, BLA), both High frequency and Low frequency rats showed increased c-Fos expression as compared with cocaine-naive rats, although High frequency rats still produced more c-Fos expression as compared with Low frequency rats.

Higher c-Fos expression in High compared with Low frequency rats is unlikely to be a general nonspecific effect 
due to a behavioral or a biochemical/metabolic bias. First, locomotor activity, expressed during this last session, did not correlate with c-Fos expression (Supplementary Table S1, Supplementary Information). Second, among other structures investigated, two could be identified in which c-Fos expression was similarly activated in Low and High frequency rats, ie, cingular cortex area 2 (CG2) and hippocampal cornu ammonis 2 (CA2) (Supplementary Information and Supplementary Figure S5). Third, brains were sampled $80 \mathrm{~min}$ after the last of the eight cocaine selfinfusions in all rats. c-Fos expression reflects strong neuronal activity about $1 \mathrm{~h}$ before sacrifice. At this time, corresponding to $20 \mathrm{~min}$ after the last infusion, considering the dynamics of self-infusions and estimated elimination of cocaine from the brain, the calculated cocaine brain levels were similarly low in the two groups (see Figure $8 \mathrm{C}$ and F).

Qualitative differences in c-Fos expression between Low and High frequency rats (Experiment 1)

Cluster analysis. The two groups showed different hierarchical organization between the five structures of interest. In the Low frequency rats, cortical structures (IL and PL) and subcortical structures (NAc core, NAc shell, and BLA) clustered separately and distantly (Figure 5a-left). NAc core and shell showed the smallest Euclidean distance. In the High frequency rats, this cortical/subcortical hierarchy was not observed; instead, PL and NAc core clustered closely together (Figure 5b-left).

Correlations. Significant correlations of c-Fos expression were observed between the five structures. However, different structures were involved depending on the experimental group. Notably, expression in PL and NAc core correlated positively in high $(R=0.91 ; p<0.05)$, but not low frequency rats. In contrast, expression in BLA and NAc core correlated positively in the low $(R=0.83$; $p<0.05$ ), but not high frequency rats (Figure $5 \mathrm{a}$ and b-right).

\section{Optogenetic Inhibition of PL Neuronal Activity in High Frequency Rats Decreases Cocaine-Induced Reinstatement; Optogenetic Activation of PL Neuronal Activity in Low frequency Rats has no Effect (Experiment 2)}

In session 27, as in session 24, rats from Experiment 2 were tested for cocaine-induced reinstatement but under PL optical stimulation.

In session 24 (Figure 6a), EYFP, ArchT and ChR2 rats differed for cocaine-induced reinstatement (Group $\times$ Hole $\times$ Dose, $\left.\mathrm{F}_{(6,63)}=2.48, p<0.05\right)$. Specifically, EYFP and ArchT (High frequency) rats did not differ, but showed a higher reinstatement than ChR2 (Low frequency) rats $(p<0.05$ for both).

In session 27, the three groups still differed for cocaineinduced reinstatement (Group effect, $\mathrm{F}_{(2,21)}=3.24, p<0.05$ ). However, ArchT (High frequency) and ChR2 (Low frequency) rats showed a similar reinstatement that was significantly lower than reinstatement by EYFP rats (High frequency) ( $p<0.05$ for both) (Figure 6b). Reinstatement was similar in session 24 and session 27 in EYFP rats as well as in ChR2 rats. Remarkably, there was a decrease of reinstatement in $\operatorname{ArchT}$ rats (Stimulation $\times$ Dose $\times$ Hole, $F_{(3,54)}=2.92$, $p<0.05]$. Thus, PL inactivation in High frequency rats decreased cocaine-induced reinstatement, while PL activation in Low frequency rats had no effect.

Optical stimulation did not alter responding during the first $50 \mathrm{~min}$ of the session, ie, during extinction, which
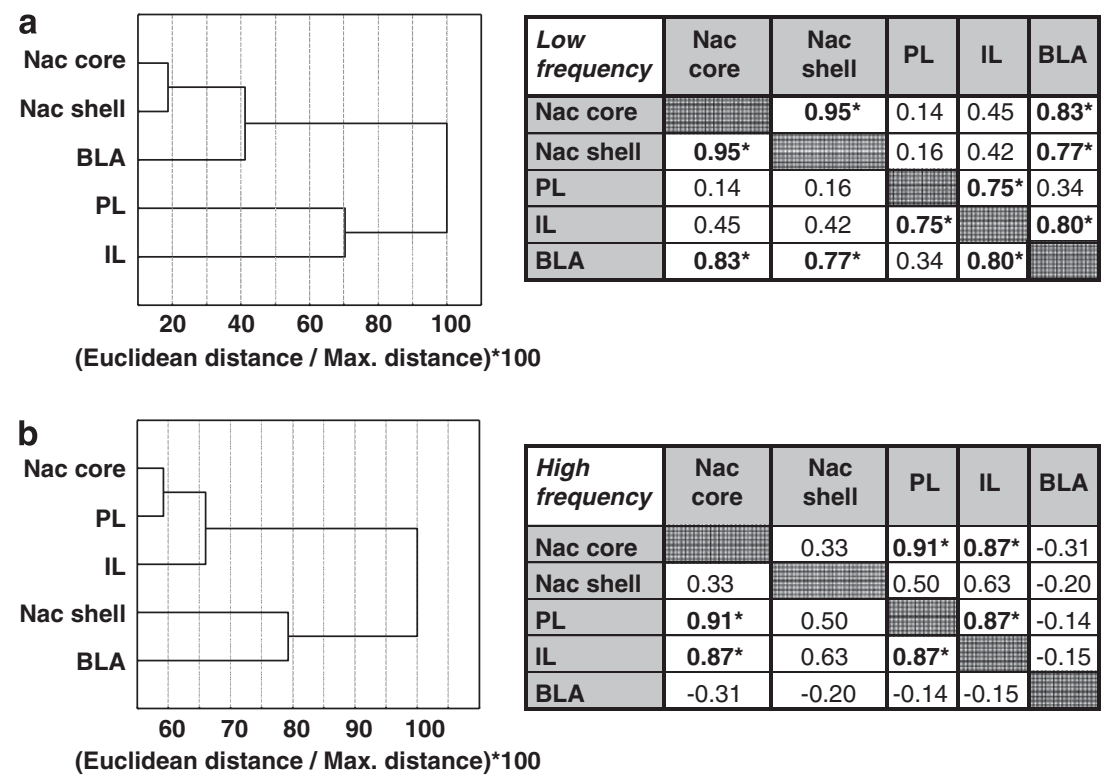

Figure 5 Cluster analysis of the hierarchical organization between the five structures of interest in the Low and High frequency rats. (a) In Low frequency rats, cortical structures (IL and PL) and subcortical structures (NAc core, NAc shell and BLA) clustered separately and distantly. NAc core and shell showed the smallest Euclidean distance. (b) In High frequency rats the cortical/subcortical hierarchy evidenced in Low frequency rats was not observed, ie, PL and NAc core clustered close together. Correlations of c-Fos expression between structures in Low (Top table) and High frequency rats (Bottom table), respectively. Different profiles of significant correlations were observed; a strong positive correlation between PL and NAc core in the High frequency group, a strong positive correlation between BLA and NAc core in the Low frequency group ( $n=6$ per group). 

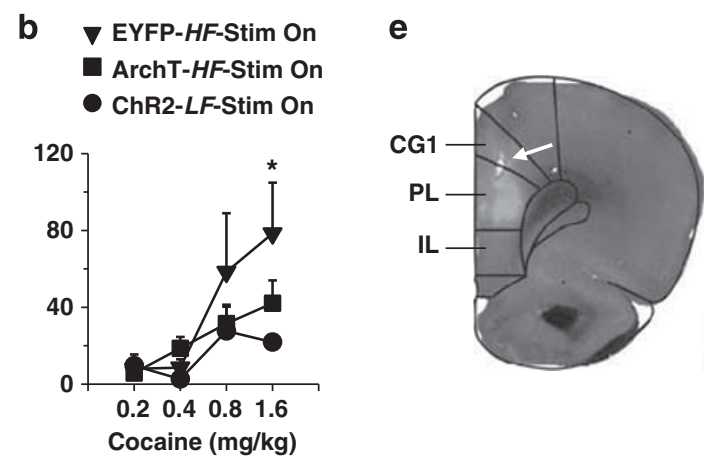

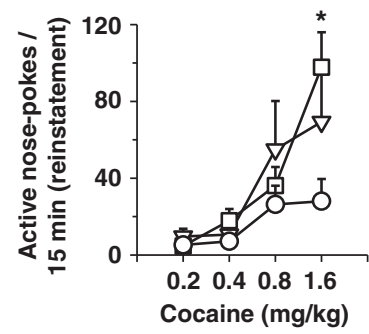

$\nabla$ EYFP-HF-Stim Off

$\square$ ArchT-HF-Stim Off

O ChR2-LF-Stim Off

Cocaine (mg/kg)

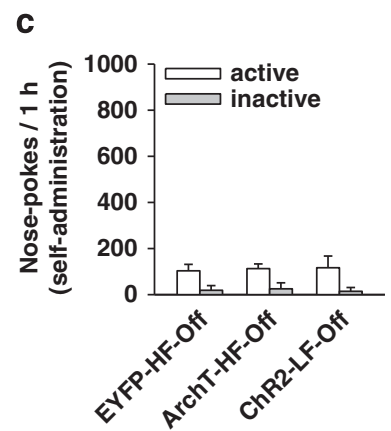

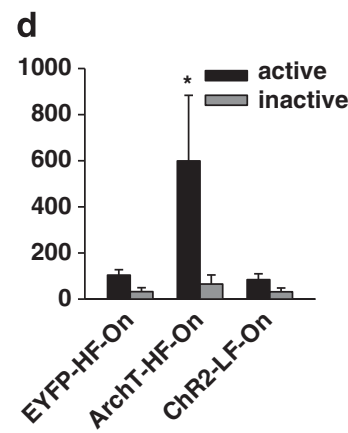

Figure 6 Effect of optical manipulation of the PL on cocaine-induced reinstatement ( $a, b)$ and cocaine self-administration (c, d). (a, b) Cocaine-induced reinstatement in EYFP, ChR2 and ArchT rats. (a) Reinstatement of extinguished behavior measured by active nose-pokes in response to non-contingent infusions of increasing doses of cocaine in absence of optical stimulation (control condition: Stim Off). EYFP and ArchT ((both High frequency (HF)) showed a comparable reinstatement that was higher as compared with ChR2 rats (Low frequency (LF)). $* p<0.05$ ArchT and EYFP as compared with ChR2. (b) Same as in panel a but during optical stimulation (Stim On). Inactivation of the PL in ArchT rats significantly decreased reinstatement while its activation in ChR2 rats was without effect. Optical stimulation did not alter the behavior of the EYFP rats. ${ }^{*} p<0.05$ EYFP as compared with ArchT and ChR2. (c, d) Cocaine selfadministration (60 min session) in High frequency (HF) (ArchT and EYFP) and Low frequency (LF) (ChR2) rats. (c) The three groups did not differ for cocaine selfadministration as measured by the total number of active nose-pokes (compared with inactive nose-pokes) over 60 min in the absence of optical stimulation (Stim Off). (d) Same as in panel c but with optical stimulation being used (Stim On). PL inactivation in High frequency rats (ArchT-HF-On) increased cocaine self-administration, while PL activation in Low frequency rats (ChR2-LF-On) had no effect. Optical stimulation did not alter behavior in the EYFP rats. *P $<0.05$ ArchT as compared with EYFP and ChR2. (e, f) Example of EYFP expression labeling and localization of optic fiber implantation in all tested rats. (e) EYFP was largely expressed in the PL. (f) Specificity of PL inactivation or activation relied on optical fiber localization. Each dot represents the tip of a fiber. CGI, cingular cortex area I; IL, infralimbic cortex; PL, prelimbic cortex.

precedes non-contingent cocaine infusions. In both session 24 (stim Off) and 27 (stim On), self-administration behavior extinguished over the $50 \mathrm{~min}$ (Hole $\times$ Time, $\mathrm{F}_{(9,189)}=2.10$, $p<0.05$; Hole $\times$ Time, $\mathrm{F}_{(9,189)}=2.55, p<0.01$, respectively) and the three experimental groups did not differ.

Optogenetic Inhibition of PL Neuronal Activity in High Frequency Rats Increased Cocaine Seeking in a Basal Self-Administration Session; Optogenetic Activation of PL Neuronal Activity in Low frequency Rats had no Effect (Experiment 2)

When optical stimulation was off, ie, in basal conditions, the three groups did not differ in total nose-pokes or discrimination (Figure 6c). Optical stimulation altered discrimination as a function of the group (Hole $\times$ Stim $\times$ Group, $\left.\mathrm{F}_{(2,22)}=2,86, p<0.05\right)$. Stimulation specifically increased active responses in the ArchT group without affecting responses in the other two groups (Figure 6d). Stimulation did not impact the total number of infusions received during the test or the mean III (Supplementary Figure S6), suggesting that the increase in active nose-pokes in ArchT rats was attributable mainly to responses during $\mathrm{TO}$ periods. Importantly, this increase was associated with an alteration in the time course of active responses (Stim $\times$ Time, $\left.\mathrm{F}_{(59,590)}=2,28, \quad p<0.0001\right)$ : stimulation did not produce an immediate increase in active nose-pokes, rather it was progressive and reached significance after $14 \mathrm{~min}$ (NK post hoc, $p<0.05$ ) (Figure $7 \mathrm{~b}$ ). The time course of infusions was not changed (Figure $7 \mathrm{~d}$ ). In comparison, optical stimulation did not alter time course of active nose-pokes (Figure 7a) or time course of infusions in ChR2 rats (Low frequency) (Figure 7c). As expected, in both $O N$ and $O F F$ stimulation conditions, over $60 \mathrm{~min}$ of self-administration, High frequency rats reached higher level of infusions than Low frequency rats (Figure 7c and d, Supplementary Figure S6A). Also, as expected, ChR2 rats (Low frequency) showed a lower III than both EYFP and ArchT rats (High frequency) (Supplementary Figure S6B).

Impact of High $v s$ Low Frequency Self-Administration on Calculated Whole-Brain Cocaine Concentrations on the 7th, 23rd and Last Sessions (Experiment 1)

Cocaine concentration in the brain was calculated in $10 \mathrm{~s}$ intervals during the 7 th self-administration session, ie, before the different TO conditions were applied, and is shown for two representative rats (rats 1 and 2), in Figure 8a 
a

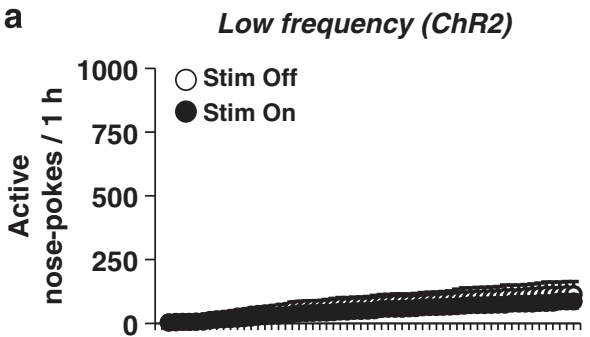

C

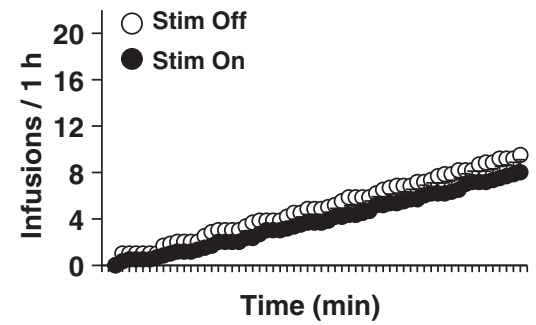

b
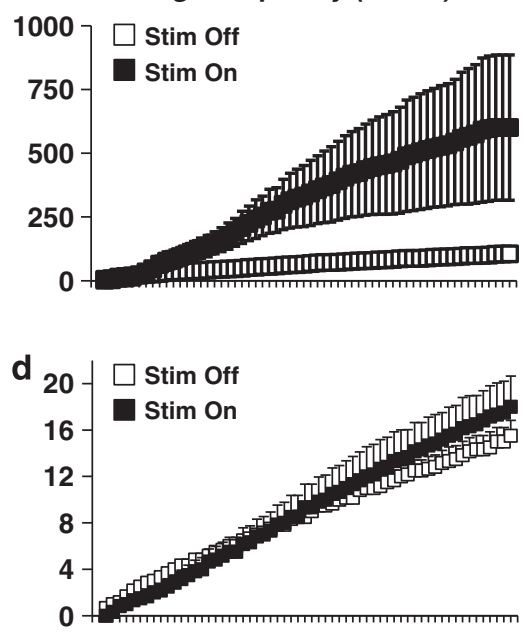

Time (min)

Figure 7 Time course of cocaine seeking (a, b) and taking (c, d) during PL optogenetic manipulation in Low (ChR2) (a-c) and High frequency rats (ArchT) (b-d). Cocaine seeking and taking are expressed by the cumulative active nose-pokes and the cumulative infusions over time (per min), respectively. (a) PL activation in Low frequency rats (ChR2) did not alter active nose-poking (Stim On as compared to Stim Off). (b) PL inactivation (ArchT) in High frequency rats increased active nose-pokes in a time-dependent manner (Stim On as compared to Stim Off) with a significant difference occurring after I 4 min. (c, d) Time course of self-administration was altered by optical stimulation neither in Low nor High frequency rats. Over 60 min of self-administration, High frequency rats self-administered more infusions than Low frequency rats, as expected.

a
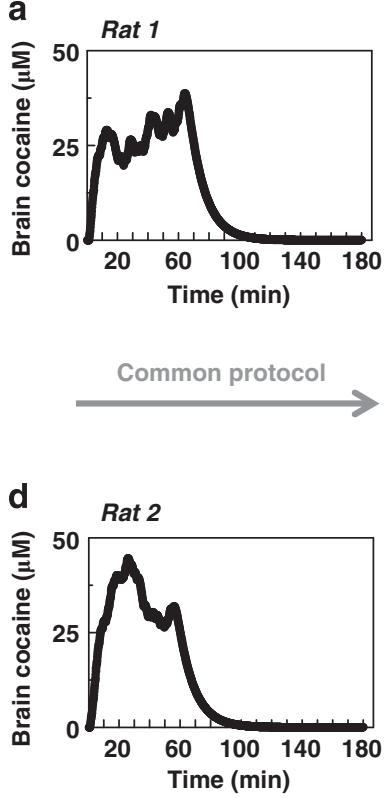

Sessions 1 to 8

Inf. max. 30 or

time max $180 \mathrm{~min}$ b
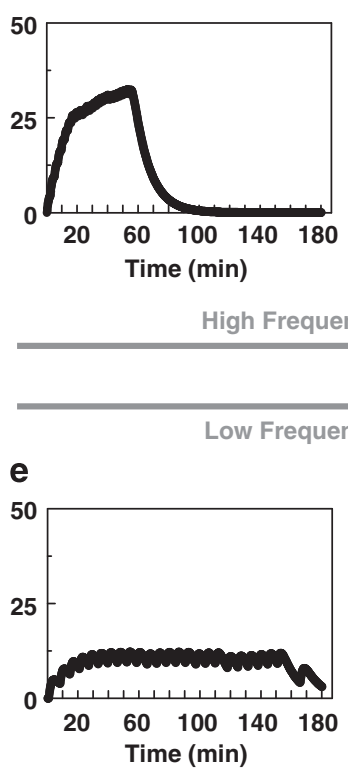

Session 9 to $28^{*}$

Inf. Max. 25 or

time max $180 \mathrm{~min}$

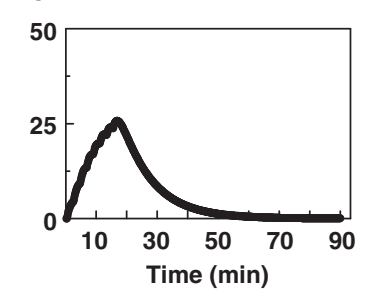

c

ncy Intake

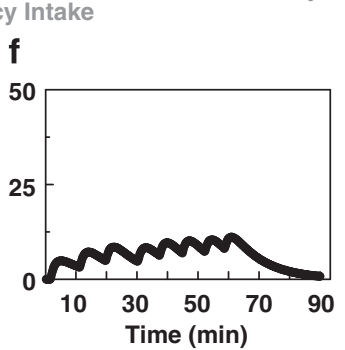

Session 29

Inf. Max. 8 or

time max $90 \mathrm{~min}$

Figure 8 Calculated brain cocaine concentrations in two representative rats of the High and Low frequency groups. Whole-brain levels of cocaine were mathematically evaluated every 10 s and are represented over session 7 (a-d), session 23 (b-e), and over the last session (c-f) for one High frequency rat (rat I, upper line) and one Low frequency rat (rat 2, lower line). Session 7 was the penultimate session of acquisition with the 40-s time-out (TO) condition for all rats. Cocaine infusions were limited to 30. Session 23 was the I5th session of the TO-specific procedure promoting High and Low frequency selfadministration; cocaine infusions were limited to 25. In both cases, the session ended after 180 min or when the maximal number of infusions allowed was reached. The last session (session 29) was the one immediately preceding sacrifice for brain sampling. Cocaine infusions were limited to eight, and session length was limited to $90 \mathrm{~min}$. For each rat, sacrifice was performed 80 min after the end of its session. Rats I and 2 showed a comparable time course of brain cocaine concentrations in session 7. In session 23 and in the last session, they showed very distinct time courses of cocaine brain concentrations. 
and $\mathrm{d}$. In this session, rats were allowed to self-administer 30 infusions over $180 \mathrm{~min}$ maximum. Considering their intake, both groups would reach within 10-15 min comparable brain concentrations of cocaine that would stabilize over $60 \mathrm{~min}$ to 30-40 $\mu$ M. Starting session 9, rats were submitted to TO conditions, which led to high and low frequency selfadministration, respectively. Here, rats were allowed to selfadminister a maximum of 25 infusions over a maximum of $180 \mathrm{~min}$. Session $23 \mathrm{rd}$ (Figure $8 \mathrm{~b}$ and e) is the 15 th session of this new condition. Rat 1 would have reached $30 \mu \mathrm{M}$ of cocaine within $15 \mathrm{~min}$ and maintained it, over $45 \mathrm{~min}$. Rat 2 would have reached as rapidly a maximum of $10 \mu \mathrm{M}$, maintained, over almost $180 \mathrm{~min}$. The same general profile was calculated based on intake during the last session before sacrifice (Figure $8 \mathrm{c}$ and $\mathrm{f}$ ). In this case, infusions were limited to 8 and the session to $90 \mathrm{~min}$.

Detailed results and statistics are provided in Supplementary Information.

\section{DISCUSSION}

These results demonstrate that manipulating the frequency of cocaine use can influence reinstatement of cocaine seeking. Our data indicate that cocaine seeking is controlled by the PL for high, but not low, frequency use. In addition, the PL can either positively or negatively control cocaine seeking, depending on the contingencies regulating the instrumental responses.

\section{PL Neuronal Activity Promotes Cocaine-Induced Reinstatement in Rats, which Self-Administered Cocaine at High Frequency}

After acquisition of cocaine self-administration, we used behavioral procedures that promoted higher or lower frequency self-administration. Rats were then tested for reinstatement of self-administration behavior induced by non-contingent infusions of cocaine. Higher frequency cocaine self-administration was associated with higher cocaine-induced reinstatement of cocaine seeking. It is unlikely that the differences observed in cocaine-induced reinstatement between High and Low frequency rats are due to behavioral biases because the number of active nosepokes was similar between the two groups.

On the basis of pharmacological (Capriles et al, 2003), lesion (Pelloux et al, 2013), and optogenetic (Stefanik et al, 2013) studies, we hypothesized that higher cocaine seeking in High frequency rats could rely on increased neuronal activity in the PL. Accordingly, optical inactivation of the PL in High frequency rats decreased cocaineinduced reinstatement of cocaine seeking to the level of Low frequency rats.

Importantly, distinct factors are known to promote relapse to cocaine seeking. They are modeled through cue-/context-, stress- and drug-induced reinstatement in rodents (Epstein et al, 2006). The neurobiological circuits supporting reinstatement differ substantially according to the triggering stimulus (Buffalari and See, 2010; Kalivas and McFarland, 2003). Our results apply to cocaine-induced reinstatement. Whether frequency of use also alters other forms of reinstatement is unknown.
High Frequency Cocaine Self-Administration Promotes Correlated c-Fos Activity in PL and NAc Core; Low Frequency Cocaine Self-Administration Promotes Correlated c-Fos Activity in BLA and NAc Core

Using c-Fos expression as a marker of neuronal activity, we investigated how frequency of cocaine self-administration altered neuronal activation in the PL and associated structures (IL, BLA, NAc core and NAc shell), forming a key network involved in cocaine-induced reinstatement (Peters et al, 2008). Cocaine-induced c-Fos expression was evaluated 80 min after High and Low frequency rats had self-administered eight cocaine infusions. c-Fos expression was higher in High frequency rats in both the PL and the four related structures. Not only were the structures of interest more intensely recruited by cocaine in High frequency rats, but they were also recruited differently. The cluster analysis, evaluating distance in the intensity of c-Fos expression between the brain structures, revealed a cortical/subcortical cleavage in the Low frequency rats, as shown by a lower distance within cortical, and within subcortical areas, as opposed to between them. A distinct functional recruitment of the circuit in the two groups was also supported by correlation analyses. Notably, in response to cocaine self-administration, strong positive correlations were observed between the BLA, the Nac shell, and the NAc core in the Low frequency rats, but not in the High frequency rats. These later showed a specific strong positive correlation between the medial prefrontal cortex, particularly the PL, and the NAc core.

Both BLA and PL projections to the NAc core might control cocaine seeking (Di Ciano and Everitt, 2004; McFarland and Kalivas, 2001; McFarland et al, 2003). However, it is likely that the underlying mechanisms could be different. Functional connectivity between the BLA and NAc core is known to be implicated in processing information about emotional or motivational significance and in mediating Pavlovian and instrumental incentive processes (Shiflett and Balleine, 2010). In contrast, connectivity between the PL and NAc core could contribute to reward-associated action selection (Mannella et al, 2013) by integrating learned contingencies that mediate instrumental performance and ultimately behavioral control.

Depending on the frequency of self-administration, cocaine might promote activation of one pathway over the other. It is unknown whether the observed c-Fos differences result from acute or repeated (during the 19 preceding sessions) cocaine self-administration at High vs Low frequency. Nevertheless, the observed differential recruitment of the circuit is likely to influence expression and control of cocaine seeking and supports the view that the PL has a more predominant role in controlling cocaine seeking in High frequency rats than in Low frequency rats. Further studies will be required to investigate the role of the BLA in cocaine seeking in High and Low frequency rats.

It has to be noted that the control rats used might be a limitation, as they were not exposed to the same extensive operant training and responding as rats trained for cocaine self-administration.

\section{PL Neuronal Activity Inhibits Cocaine Self-Administration}

In High frequency rats, the same inactivation that decreased cocaine-induced reinstatement produced a strong increase 
in cocaine self-administration. Importantly, the increased seeking in response to PL optical inactivation only occurred after a few cocaine self-infusions and was mainly during the time-out periods. This suggests that the PL is recruited after a given load of cocaine to engage control on non-adapted operant responses. In the case of optical manipulation (ie, PL inactivation), this recruitment would not occur and control would be lost.

Whether this inhibitory control exerted by the PL on selfadministration results from previous repeated exposure to High frequency cocaine is questionable. Indeed, it cannot be ruled out that it is an acute involvement of the PL due to High frequency access to cocaine that would not occur in Low frequency access, for example. In other words, the influence exerted by the PL in High frequency rats on selfadministration could be different from the one exerted during reinstatement.

However, although paradoxical, the opposite effect of PL inactivation on reinstatement and self-administration fits with a role of the PL in coordinating the acquired contingencies that mediate operant responses. Inactivation of the PL would compromise retrieval of the operant response after extinction (ie, decrease reinstatement), but would also dysregulate reinforced instrumental responses (ie, increase self-administration), compromising control of acquired contingencies such as time-out, FR, and so on. Accordingly, this dysregulation in operant responding produced by PL inactivation is also observed for responses reinforced by natural reinforcers. Jonkman et al (2009) investigated the involvement of the PL in acquisition of instrumental responding for a food reinforcer, under fixed interval schedules. Inactivation of the PL by post-session infusion of a protein synthesis inhibitor did not affect any measure of acquisition, but strongly increased established responding, leading the authors to conclude that the PL might mediate a form of inhibitory response control.

Recently, this paradoxical opposite effect of PL inactivation was also observed for alcohol. Pharmacological inactivation of the PL increased, or reduced, nose-poking for alcohol, depending on whether behavior was reinforced by the drug or not (Willcocks and McNally, 2013).

The ability of the PL to exert inhibitory control on cocaine seeking is also supported by the recent work by Chen et al (2013). Cocaine seeking was suppressed by footshock in some rats. Optical inactivation of the PL abolished this foot-shock-induced decrease in cocaine seeking, an effect that was not observed by Pelloux et al (2013) after PL lesions. Considering that PL controls learned instrumental responses but not their acquisition (Jonkman et al, 2009), a main procedural difference could explain this discrepancy. Both studies used a seeking-taking chain procedure, with the seeking response being randomly punished by an electric foot-shock. In Chen et al (2013), the taking lever and cocaine remained accessible. Suppression of seeking responding by shock would rely on a conflicting situation between punishment and cocaine reinforcement. Alternatively, in Pelloux et al (2013), suppression of responding by shock would rely on new learning. Indeed the seeking response is not only punished by a shock but also extinguished because it is not reinforced anymore by the occurrence of the taking lever.
Although optical inactivation of the PL abolished a footshock-induced decrease in cocaine seeking, Chen et al (2013) also showed that it did not alter basal cocaine selfadministration behavior, a discrepancy with our study. However, in Chen et al (2013), optical stimulation is only applied during presentation of the seeking lever. Thus, the PL can be activated when cocaine is delivered, allowing its recruitment to control operant responding. Alternatively, this discrepancy could result from a ceiling effect compromising the expression of increased seeking. Indeed, in Chen et al (2013), control rats already self-administered the maximal number of infusions with a very short inter-press interval (around $35 \mathrm{~s}$ ).

\section{PL Neuronal Activity does not Control Cocaine Seeking in Low frequency Rats}

While PL inactivation can promote dysregulated cocaine seeking (present study, Chen et al, 2013), PL activation can produce the opposite effect, ie, inhibit responses in rats maintaining drug-seeking responses despite electric footshocks (Chen et al, 2013). This dual effect reveals that control of behavior by the PL can be modulated bidirectionally. It also links regulated/deregulated responding with hypoactivity/hyperactivity in the PL, respectively. In the present study, optical activation of the PL in Low frequency rats did not alter cocaine-induced reinstatement or cocaine self-administration. This suggests that the PL was not recruited to control cocaine seeking in Low frequency rats.

It was beyond our experimental hypothesis to apply optical inhibition of the PL in Low frequency rats. Therefore, we cannot rule out that such inhibition could affect either self-administration or reinstatement. As well we cannot foresee the behavioral consequences of PL activation in High frequency rats.

\section{High and Low frequency Rats: Distinct Pharmacological Profiles}

Calculation of brain cocaine levels supports that differences in frequency of use imply differences in both cocaine dynamics (time to peak) and cocaine peak level. High frequency self-administration achieves faster, higher and shorter lasting brain cocaine levels. In the present selfadministration conditions, High frequency rats reached a theoretical maximal brain concentration of cocaine that is approximately three times higher than the one reached by Low frequency rats. Cocaine concentration reached $10 \mu \mathrm{M}$ in 20 min in the Low frequency rats, as compared with fewer than $10 \mathrm{~min}$ in High frequency rats. Finally, as the maximal number of self-infusions is limited, cocaine would be present in the brain three times longer in Low frequency rats than in High frequency rats.

Whether the behavioral differences between High and Low frequency rats rely on differences in cocaine dynamics, peak brain level, or an interaction of both, cannot be disentangled based on the present procedure. Nevertheless, several observations support the view that peak level and temporal dynamic interact to recruit specific circuits involved in promoting/inhibiting cocaine seeking. 
Indeed, differences in peak levels reached could explain the higher c-Fos activation shown by High frequency rats in the five structures of interest. However, qualitative differences in c-Fos activation between these structures, together with similar c-Fos activation in High and Low frequency rats for other brain structures (ie, CG2 and CA2-see Supplementary Information), suggest that the dynamics of cocaine delivery also have a role in the observed biological and behavioral effects.

Critically, modulating dynamics alters cocaine-induced reinstatement only if cocaine intake is high. Shortening the time to peak cocaine brain levels (through narrow manipulation of the rate of infusion from 5 to $90 \mathrm{~s}$ ), without altering the peak level achieved, provokes sensitization of the psychomotor and reinforcing effects of psychostimulants and produces specific and relevant neurobiological adaptations (Ferrario et al, 2008; Liu et al, 2005a, 2005b; Samaha and Robinson, 2005; Samaha et al, 2002, 2004). However, it is associated with increased cocaine-induced reinstatement only in rats that have had long $(6 \mathrm{~h})$, but not short $(1 \mathrm{~h})$, daily access to the drug (Crombag et al, 2008; Wakabayashi et al, 2010). In addition, fast delivery rate is necessary to observe high frequency use (escalation) in the 6-h rats (Wakabayashi et al, 2010), supporting that the two factors, ie, high reinstatement and high frequency use, are related. Finally, protocols leading to repeated transiently high plasma levels of cocaine increase motivation for cocaine, supporting that frequency of cocaine use can impact its psychopharmacological consequences (Zimmer et al, 2012).

\section{CONCLUSIONS}

In a multi-symptomatic model of cocaine addiction in the rat (Deroche-Gamonet and Piazza, 2014; Deroche-Gamonet et al, 2004), we observed both High frequency self-administration and high cocaine-induced reinstatement in about $20 \%$ of addiction-vulnerable rats. These features are present both after early and late cocaine training (Belin et al, 2009). However, after late training, these rats also express addiction-like behaviors, ie, dysregulated cocaine seeking. They seek drug, although it is signaled as unavailable. They also seek and take cocaine despite punishment by electric foot-shocks. Their early behavioral profile is compatible with an involvement of the PL in controlling cocaine seeking, while their late profile is not, at least in terms of PL functionality after early training. How a greater role of PL in controlling responses for cocaine after early training might lead to cocaine addiction behavior is unknown and remains to be investigated. However, altogether our data suggest that high frequency use could be a risk factor for increased cocaine-induced reinstatement of cocaine seeking and addiction, and the PL is a target of interest in regulating $v s$ dysregulating operant responses for cocaine.

\section{FUNDING AND DISCLOSURE}

This work was supported by INSERM, Université of Bordeaux Segalen and Région Aquitaine, by ANR-2010BLAN-1442-01, ANR-10-EQX-008-1 and LABEX BRAIN ANR-10-LABX-43 grants to CH and VDG. EMG was supported by a 'Sara Borrell' post-doctoral fellowship (CD07/00244) from the Spanish 'Instituto de Salud Carlos
III'. JC was supported by an AXA doctoral fellowship. The authors declare no conflict of interest.

\section{ACKNOWLEDGEMENTS}

The microscopy was done in the Bordeaux Imaging Center of the University of Bordeaux Segalen. The help of Sébastien Marais is acknowledged. We thank Dr R. Rozeske and Robyn St Laurent for precious help with editing. We thank Dr D Belin for discussion of an early version of the manuscript.

\section{REFERENCES}

Ball KT, Slane M (2012). Differential involvement of prelimbic and infralimbic medial prefrontal cortex in discrete cue-induced reinstatement of 3,4-methylenedioxymethamphetamine (MDMA; ecstasy) seeking in rats. Psychopharmacology 224: 377-385.

Belin D, Balado E, Piazza PV, Deroche-Gamonet V (2009). Pattern of intake and drug craving predict the development of cocaine addiction-like behavior in rats. Biol Psychiatry 65: 863-868.

Bossert JM, Marchant NJ, Calu DJ, Shaham Y (2013). The reinstatement model of drug relapse: recent neurobiological findings, emerging research topics, and translational research. Psychopharmacology 229: 453-476.

Buffalari DM, See RE (2010). Amygdala mechanisms of Pavlovian psychostimulant conditioning and relapse. Curr Top Behav Neurosci 3: 73-99.

Capriles N, Rodaros D, Sorge RE, Stewart J (2003). A role for the prefrontal cortex in stress- and cocaine-induced reinstatement of cocaine seeking in rats. Psychopharmacology 168: 66-74.

Chen BT, Yau H-J, Hatch C, Kusumoto-Yoshida I, Cho SL, Hopf FW et al (2013). Rescuing cocaine-induced prefrontal cortex hypoactivity prevents compulsive cocaine seeking. Nature 496: 359-362.

Di Ciano P, Everitt BJ (2004). Direct interactions between the basolateral amygdala and nucleus accumbens core underlie cocaine-seeking behavior by rats. J Neurosci 24: 7167-7173.

Crombag HS, Ferrario CR, Robinson TE (2008). The rate of intravenous cocaine or amphetamine delivery does not influence drug-taking and drug-seeking behavior in rats. Pharmacol Biochem Behav 90: 797-804.

Dackis CA, O'Brien CP (2001). Cocaine dependence: a disease of the brain's reward centers. J Subst Abuse Treat 21: 111-117.

Deroche-Gamonet V, Belin D, Piazza PV (2004). Evidence for addiction-like behavior in the rat. Science 305: 1014-1017.

Deroche-Gamonet V, Piazza PV (2014). Psychobiology of cocaine addiction: Contribution of a multi-symptomatic animal model of loss of control. Neuropharmacology 76(Pt B): 437-449.

Epstein DH, Preston KL, Stewart J, Shaham Y (2006). Toward a model of drug relapse: an assessment of the validity of the reinstatement procedure. Psychopharmacology 189: 1-16.

Ferrario CR, Shou M, Samaha AN, Watson CJ, Kennedy RT, Robinson TE (2008). The rate of intravenous cocaine administration alters c-fos mRNA expression and the temporal dynamics of dopamine, but not glutamate, overflow in the striatum. Brain Res 1209: 151-156.

Gawin FH (1991). Cocaine addiction: psychology and neurophysiology. Science 251: 1580-1586.

Gawin FH, Khalsa-Denison ME (1996). Is craving mood-driven or self-propelled? Sensitization and 'street' stimulant addiction. Nida Res Monogr 163: 224-250.

Gawin FH, Kleber HD (1988). Evolving conceptualizations of cocaine dependence. Yale J Biol Med 61: 123-136.

Goldstein RZ, Volkow ND (2011). Dysfunction of the prefrontal cortex in addiction: neuroimaging findings and clinical implications. Nat Rev Neurosci 12: 652-669. 
Herry C, Mons N (2004). Resistance to extinction is associated with impaired immediate early gene induction in medial prefrontal cortex and amygdala. Eur J Neurosci 20: 781-790.

Jaffe JH, Cascella NG, Kumor KM, Sherer MA (1989). Cocaineinduced cocaine craving. Psychopharmacology 97: 59-64.

Jonkman S, Mar AC, Dickinson A, Robbins TW, Everitt BJ (2009). The rat prelimbic cortex mediates inhibitory response control but not the consolidation of instrumental learning. Behav Neurosci 123: 875-885.

Kalivas PW, McFarland K (2003). Brain circuitry and the reinstatement of cocaine-seeking behavior. Psychopharmacology 168: 44-56.

LaLumiere RT, Smith KC, Kalivas PW (2012). Neural circuit competition in cocaine-seeking: roles of the infralimbic cortex and nucleus accumbens shell. Eur J Neurosci 35: 614-622.

Liu Y, Roberts DCS, Morgan D (2005a). Sensitization of the reinforcing effects of self-administered cocaine in rats: effects of dose and intravenous injection speed. Eur J Neurosci 22: 195-200.

Liu Y, Roberts DCS, Morgan D (2005b). Effects of extended-access self-administration and deprivation on breakpoints maintained by cocaine in rats. Psychopharmacology 179: 644-651.

Mannella F, Gurney K, Baldassarre G (2013). The nucleus accumbens as a nexus between values and goals in goal-directed behavior: a review and a new hypothesis. Front Behav Neurosci 7: 135.

McFarland K, Kalivas PW (2001). The circuitry mediating cocaineinduced reinstatement of drug-seeking behavior. J Neurosci 21: 8655-8663.

McFarland K, Lapish CC, Kalivas PW (2003). Prefrontal glutamate release into the core of the nucleus accumbens mediates cocaineinduced reinstatement of drug-seeking behavior. J Neurosci 23: 3531-3537.

McLaughlin J, See RE (2003). Selective inactivation of the dorsomedial prefrontal cortex and the basolateral amygdala attenuates conditioned-cued reinstatement of extinguished cocaine-seeking behavior in rats. Psychopharmacology 168: 57-65.

Mihindou C, Guillem K, Navailles S, Vouillac C, Ahmed SH (2013). Discriminative inhibitory control of cocaine seeking involves the prelimbic prefrontal cortex. Biol Psychiatry 73: 271-279.

Paxinos G, Watson C (2005). The Rat Brain In Stereotaxic Coordinates. 5th edn, Elsevier Academic Press: Amsterdam, The Netherlands.
Pelloux Y, Murray JE, Everitt BJ (2013). Differential roles of the prefrontal cortical subregions and basolateral amygdala in compulsive cocaine seeking and relapse after voluntary abstinence in rats. Eur J Neurosci 38: 3018-3026.

Peters J, LaLumiere RT, Kalivas PW (2008). Infralimbic prefrontal cortex is responsible for inhibiting cocaine seeking in extinguished rats. J Neurosci 28: 6046-6053.

Samaha A-N, Li Y, Robinson TE (2002). The rate of intravenous cocaine administration determines susceptibility to sensitization. J Neurosci 22: 3244-3250.

Samaha A-N, Mallet N, Ferguson SM, Gonon F, Robinson TE (2004). The rate of cocaine administration alters gene regulation and behavioral plasticity: implications for addiction. J Neurosci 24: 6362-6370.

Samaha A-N, Robinson TE (2005). Why does the rapid delivery of drugs to the brain promote addiction? Trends Pharmacol Sci 26: 82-87.

Shiflett MW, Balleine BW (2010). At the limbic-motor interface: disconnection of basolateral amygdala from nucleus accumbens core and shell reveals dissociable components of incentive motivation. Eur J Neurosci 32: 1735-1743.

Stefanik MT, Moussawi K, Kupchik YM, Smith KC, Miller RL, Huff ML et al (2013). Optogenetic inhibition of cocaine seeking in rats. Addict Biol 18: 50-53.

Vertes RP (2006). Interactions among the medial prefrontal cortex, hippocampus and midline thalamus in emotional and cognitive processing in the rat. Neuroscience 142: 1-20.

Wakabayashi KT, Weiss MJ, Pickup KN, Robinson TE (2010). Rats markedly escalate their intake and show a persistent susceptibility to reinstatement only when cocaine is injected rapidly. J Neurosci 30: 11346-11355.

Willcocks AL, McNally GP (2013). The role of medial prefrontal cortex in extinction and reinstatement of alcohol-seeking in rats. Eur J Neurosci 37: 259-268.

Zimmer BA, Dobrin CV, Roberts DCS (2011). Brain-cocaine concentrations determine the dose self-administered by rats on a novel behaviorally dependent dosing schedule. Neuropsychopharmacol 36: 2741-2749.

Zimmer BA, Oleson EB, Roberts DC (2012). The motivation to selfadminister is increased after a history of spiking brain levels of cocaine. Neuropsychopharmacol 37: 1901-1910.

Supplementary Information accompanies the paper on the Neuropsychopharmacology website (http://www.nature.com/npp) 